\title{
21. Troubles trophiques
}

\section{Congrès JASFGG 2014, Paris, France}

(C) Lavoisier SAS 2014

\section{P6.21-44}

\section{La neuropathie périphérique sensorielle des membres inférieurs augmente le risque d'escarre du talon chez les octogénaires}

\author{
M.-L. Gaubert-Dahan ${ }^{1,2, *}$, K. Castro-Lionard ${ }^{3}$, M.-A. Blanchon ${ }^{3}$, \\ B. Fromy ${ }^{2}$ \\ ${ }^{1}$ Gériatrie, APHP hôpital Bretonneau, Paris cedex $18{ }^{2} U M R$ CNRS \\ 5305, Université Claude Bernard Lyon 1, Lyon \\ ${ }^{3}$ Gériatrie, CHU, Saint-Étienne, France
}

Introduction : l'escarre est le résultat d'une compression prolongée des tissus entre les saillies osseuses et le plan de contact sur lequel repose le sujet immobilisé. La compression des tissus entraîne ischémie et nécrose tissulaires. Il existe au niveau cutané un couplage entre mécanosensibilité et vasodilatation qui retarde l'ischémie et protège donc la peau. Avec le vieillissement, ce réflexe neuro-vasculaire est altéré et en cas de neuropathie sensorielle, il est aboli. Les sujets âgés et en particulier ceux atteints de neuropathie sensorielle semblent donc particulièrement à risque d'escarre.

Objectifs : établir une corrélation entre neuropathie périphérique sensorielle des membres inférieurs et escarre talonnière.
Méthodes : les patients hospitalisés en Soins de Suite et Réadaptation gériatriques à Paris et Saint-Étienne ont été inclus de mars 2009 à juin 2010. Les patients ayant un Mini-Mental State Examination inférieur à 10/30 ont été exclus et de même les patients hémiplégiques ou paraplégiques. L'existence d'une escarre talonnière était notée à l'admission ainsi que son stade. La sévérité d'une neuropathie périphérique sensorielle était évaluée en utilisant les scores NSS (Neuropathy Symptom Score) et NDS (Neuropathy Disability Score) : légère (NSS $<5$ ou $\mathrm{NDS}<6$ ), moyenne (NSS=5-6 ou NDS=6-16) ou sévère $(\mathrm{NSS}>6$ ou NDS $>16$ ).

Résultats : parmi les 210 patients inclus (âge moyen $85 \pm 6$ ans, $75 \%$ de femmes), 26 patients avaient une escarre talonnière (stade $1: 13$, stade $2: 7$, stade $3: 4$ et stade $4: 2$ ). Parmi les 46 patients avec une neuropathie périphérique légère, deux avaient une escarre ; parmi les 121 patients avec une neuropathie moyenne, 13 avaient une escarre; et parmi les 43 patients avec une neuropathie sévère, 11 avaient une escarre. Le stade de l'escarre talonnière était corrélé à la sévérité de la neuropathie périphérique (Pearson 0,$22 ; p=0,001$ ).

Conclusion : La présence d'une neuropathie périphérique sensorielle des membres inférieurs augmente l'incidence et la sévérité d'escarre talonnière.

Mots clés : Escarre - Neuropathie

Congrès JASFGG 2014, Paris, France $(\triangle)$

e-mail : info@jasfgg2014.com 